\title{
Biópsia óssea na DRC
}

Bone biopsy in chronic kidney disease

\section{Autores:}

Fellype Carvalho

Barreto

Leandro Júnior Lucca
1 A biópsia óssea com dupla marcação pela tetraciclina é o único método capaz de diagnosticar o tipo histológico da doença óssea na doença renal crônica (DRC) (Evidência).

2 Em pacientes com DRC estágio III a VD, a biópsia óssea deve ser considerada principalmente nas seguintes condições: fraturas atraumáticas, dor óssea persistente, hipofosfatemia e/ou hipercalcemia não explicadas, suspeita de intoxicação alumínica, antes da paratireoidectomia e antes de terapia com bisfosfonatos (Opinião).

\section{Racional}

Recentemente, o Kidney Disease Improving Global Outcomes (KDIGO) reuniu as alterações do metabolismo mineral, da estrutura óssea e da calcificação extraesquelética sob a denominação de distúrbio mineral e ósseo da doença renal crônica (DMO-DRC). Dessa forma, o termo osteodistrofia renal (OR) passou a designar exclusivamente as alterações de morfologia óssea presentes em pacientes com DRC. ${ }^{1}$ A biópsia óssea com dupla marcação pela tetraciclina, seguida de análise histomorfométrica, é o padrão-ouro para o diagnóstico da OR. ${ }^{1,2}$ A biópsia óssea fornece informações sobre a remodelação, mineralização e volume ósseos, o que ajuda na avaliação da qualidade óssea assim como na fisiopatologia subjacente. Todavia, por se tratar de um método invasivo, de alto custo e que requer centros especializados para sua realização, a biópsia óssea não é recomendada como parte da avaliação de rotina na DRC. ${ }^{3}$ No entanto, em algumas situações clínicas específicas, a realização da biópsia óssea deve ser considerada em pacientes com DRC estágio III a V D: fraturas atraumáticas, dor óssea persistente, hipofosfatemia e/ou hipercalcemia não explicadas, suspeita de intoxicação alumínica, antes da paratireoidectomia e antes de terapia com bisfosfonatos. ${ }^{4}$

A marcação prévia do tecido ósseo pela tetraciclina se faz necessária nos pacientes que serão submetidos à biópsia. A dose utilizada de cloridrato de tetraciclina é de $20 \mathrm{mg} / \mathrm{kg} / \mathrm{dia}$ por 3 dias consecutivos, em dois períodos distintos, separados por um intervalo de 10 dias, quando a droga é descontinuada. A biópsia óssea deve ser realizada até 5 dias após o segundo período de tomada da tetraciclina. A biópsia óssea transilíaca realizada com trefina fornece amostra de osso ilíaco composto por uma área interna intacta de osso trabecular delimitado por duas corticais. O fragmento ósseo deve ser conservado em solução de álcool etílico a $70 \%$, em frasco de vidro protegido da luz. As complicações relacionadas à biópsia óssea são raras. As mais frequentes são: dor, hematoma e infecção local e, raramente, neuropatia. De acordo com um estudo multicêntrico, a incidência global de complicação após biópsia óssea transilíaca foi de $0,7 \% .^{5}$

\section{REFERÊnCIAS}

1. Moe S, Drueke T, Cunningham J et al. Kidney Disease: Improving Global Outcomes (KDIGO). Definition, evaluation, and classification of renal osteodystrophy: a position statement from Kidney Disease: Improving Global Outcomes (KDIGO). Kidney Int 2006; 69:1945-53.

2. Malluche HH, Langub MC, MonierFaugere MC. The role of bone biopsy in clinical practice and research. Kidney Int 1999; 73:S20-5. 
3. Martin KJ, Olgaard K, Coburn JW et al. Diagnosis, assessment, and treatment of bone turnover abnormalities in renal osteodystrophy. Am J Kidney Dis 2004; 43:558-65.

4. Kidney Disease: Improving Global Outcomes (KDIGO). CKD-MDB Work group. Clinical practice guideline for the diagnosis, evaluation, prevention, and treatment of chronic kidney disease and bone disorder (CKD-MDB). Kidney Int Suppl 2009;113:S1-S30.

5. Rao SD, Matkovic V, Duncan H. Transiliac bone biopsy. Complications and diagnostic value. Henry Ford Hosp Med J 1980;28:112-5. 